\title{
A Case of Senile Vaginitis Treated by Acupuncture
}

\author{
Qingqing Guo1, Gaiqin Yang1,2* \\ ${ }^{1}$ Shaanxi University of Traditional Chinese Medicine, Xianyang 712046, Shaanxi Province, China \\ ${ }^{2}$ Shaanxi Provincial Hospital of Traditional Chinese Medicine, Xi'an 710003, Shaanxi Province, China \\ *Corresponding author: Gaiqin Yang, 1335945388@qq.com
}

\begin{abstract}
The article records details of the treatment process and effect of an elderly patient with senile vaginitis, where needling, a method of Chinese Traditional Medicine (TCM) method, was applied mainly, combined with oral administration of western medicine. It then analyses this disease from prospective of TCM. In the end, it concludes that no matter traditional needling method or traditional needling method combined with moxibustion, it has achieved a satisfied treatment effect while applying needling on chosen acupuncture points, so it is worthy being further popularized.
\end{abstract}

Keywords: Senile vaginitis; Baliao point; Acupuncture therapy; Proved case

Publication date: July 2021; Online publication: July 31, 2021

\section{Introduction}

Senile vaginitis (SV) is one of the common diseases of elderly women whose main manifestations include vulvar burning, itching, and increased vaginal discharge. Moreover, the disease is highly recurrent and difficult to cure. At present, the efficacy of traditional Chinese medicine (TCM) in the treatment of SV has been clinically recognized. A case of senile vaginitis with burning pain in the vulva as the main manifestation is as follows.

The 60 year old female patient was seen by the doctor on March 18, 2021. She complained of intermittent burning pain of the vulva for more than 3 months, which has aggravated for more than one month. The patient experienced intermittent burning pain of the vulva without obvious cause more than three months ago, and had significant discomfort when urinating. The patient claimed that she didn't pay much attention to it, and she took Sophora flavescens gel for soothing her private part. The burning sensation of the vulva was slightly reduced, and the symptoms resurfaced after stopping the drug. There was no obvious cause before 1 month, and the vulvar discomfort deteriorated with more vaginal discharge, which is yellow and sticky. She often felt bitterness in the mouth, and had dry mouth, heavy bowel movements, obesity, poor sleep, and dreaminess. Her tongue was red and crimson, with yellow and greasy coating, and the pulse string was slippery. According to Western medicine diagnosis, she had senile vaginitis, whereas according to Chinese medicine diagnosis, she takes the disease with the symptoms relating to damp-heat. The main treatment indicated was acupuncture, and the method was meant to clear heat and reduce dampness. From the aspect of Western medicine, the patient was given mainly oral metronidazole, 400mg/time, 2 times/day. The acupuncture points involved are Baihui and Sanyinjiao. For supine position, Dahe, Qugu, Zhongji, Guanyuan, Ligou, and Yinlingquan were used. For prone position, Baliao point, Yaoyangguan, and Shenshu were used. Firstly, the patient took the supine position to exposes the skin of the lower abdomen and both lower limbs. Routine disinfection was performed at the acupoints. A $0.25 \times 40 \mathrm{~mm}$ milli-needle is used for acupuncture. The patient felt that the needle sensation radiates to the perineum to a degree. After the acupuncture has gained Qi, an electro-acupuncture treatment instrument was used to connect the positive and negative poles of both sides; continuous wave with a frequency 30-50 
$\mathrm{HZ}$ was chosen. Needle was applied in $15 \mathrm{~min}$, once every other day. Secondly, the patient took the prone position, exposing the skin of the sacrococcyx and both lower limbs. The Balio point was acupunctured with a $0.25 \times 75 \mathrm{~mm}$ milli-needle, which was pointed towards the perineum. The lifting and cutting method were applied. Electroacupuncture was connected to both sides of Shenshushu, and the rest of the operation was the same as before. This was applied once every other day, and 10 times were considered a course of treatment. After two courses of treatment, the patient felt that the pain of vulvar burning was significantly reduced, the amount and color of the vulva were reduced compared with before, and the frequency of repeated symptoms was reduced. The patient's symptoms have improved significantly, and the patient was instructed to wash the vulva with appropriate frequency, avoid unclean sex, and take fatty and sugary food with caution.

Modern medicine believes that the onset of SV ${ }^{[1]}$ is closely related to the decline of female ovarian function and vaginal self-regulation dysfunction, which gives rise to invasion of pathogens and disease development. In TCM, there is no corresponding terminology for this disease. However, according to the description of the related symptoms of this disease, it can be related to "carrying down disease" and "yin itching" ${ }^{[2]}$. Physicians of the past believed that the occurrence of collateral diseases was related to windcold entry into the collaterals, exogenous dampness and heat, phlegm dampness, and liver, spleen and kidney dysfunction. The occurrence of itching is related to insect erosion, yin deficiency, dampness and heat. In general, the disease is closely related to the kidney, liver, and spleen, and is also related to kidney Qi, Tiangui, and Renmai. Therefore, the TCM treatment of this disease is mainly based on Tiaobuchongren [3]; on this basis, the treatment is based on syndrome differentiation, and the corresponding treatment rules are given. This case is based on the method of clearing heat and removing dampness and promoting $q i$. Among them, the acupuncture points are mainly the bladder meridian, Renmai, and kidney meridian, and the application of manipulation and the effect of electroacupuncture are not very important. First take the Baihui, use the method of reducing to promote the use of, the second three Yinjiao is the meeting of the Zusanyin Meridian, which mainly treats the birth of a child through the meridian, regulate the liver, spleen and kidney meridians, there is a saying in "Qian Jin Fang": "...the woman leaks the scarlet and white blood...." Sanyinjiao is recognized as the key to gynecological diseases, Dahe nerve point is at the central pole, near the uterus, caused by the kidney meridian Qi. Acupuncture and Moxibustion Dacheng pointed out that "the master is vain... the woman is red." Its "hehe" potential combined with the continuous stimulation using electric acupuncture has therapeutic effect. Qugu, Zhongji, and Guanyuan Renmai points, which harmonize Chongren, in which the Renmai and foot Jueyin liver meridian intersect at Qugu, which promotes Qi and soothes the liver while also benefiting the Chongren power. Zhongji Guanyuan Zusan acupoints at the intersection of the Yin Meridian and Ren Vessel have the functions of regulating the liver, spleen and kidney, and regulating and regulating Chong Ren. Applying acupuncture at Baliao acupoints, combined with the lifting and removing method, make the reconciliation and Chong Ren very effective. Yaoyangguan and Shenshu play the role of replenishing the kidney Qi to consolidate the belt. Perineal Lingquan foot Taiyin spleen meridian combines points. "Combining the main body against the Qi and venting the Qi" is a must-chosen point for Qi lin. It is the evidence for the treatment of this medical record, which is used to promote dampness, and treat the yin pain of this disease. Yin points in the liver meridian between the lines, where "yin governs body heat," is used to relieve heat. Ligou is the collateral point of the liver meridian of foot Jueyin. According to Acupuncture and Moxibustion Dacheng, "the main colic...the woman under the red and white belt...," the yin organs of the liver meridians, and the effective points for stopping itching not only relieve the heat of the liver meridian, but also replenish liver blood to stop itching. The Baliao acupoint has a significant clinical effect on the treatment of this disease. The clinical treatment of such diseases is not only the use of acupuncture alone, but also different therapies to treat different symptoms caused by different syndromes. For example, warm acupuncture and ginger 
moxibustion, which should be adjusted accordingly and maneuvered with the method of lifting and interpolation, are used to treat deficiency-cold diseases. Some scholars believe that the clinical acupoint selection of this point is not uniform and difficult, and the operation is inconvenient, so they proposed the positioning method of "Xin Ba Liao Point." With the tailbone as the body surface mark, "lower iliac" is selected and extended slightly from bottom to top in turn; each point is separated by a cross-pointing yang. This "Xia Li" corresponds to Huiyang acupoint in our traditional sense. The bladder meridian of the left and right feet of Huiyang intersects with the governor channel to supplement the deficiency of the yang and cure the yin and dampness syndrome. Therefore, regardless of whether it is traditional acupuncture or combined moxibustion, the combined acupuncture method of selecting different acupoints has achieved satisfactory results in the clinical treatment of this disease, so it is worthy of further promotion and application.

\section{Disclosure statement}

The author declares no conflict of interest.

\section{References}

[1] Zhou HJ, 2014, Clinical Study on the Treatment of Senile Vaginitis of Liver and Kidney Yin Deficiency with Liuwei Dihuang Prescription Combined with Baofukang Suppository. Shandong University of Traditional Chinese Medicine.

[2] You XX, Su ZJ, Pei J, 2017, Lu's Acupuncture Treatment of Senile Vaginitis. Shanghai Journal of Acupuncture, 36(8): 1004-1007.

[3] Zhang HF, 2018, Analysis of the Characteristics of the Ancient Clinical Application of the External Treatment of Taking the Disease. Shandong University of Traditional Chinese Medicine. 